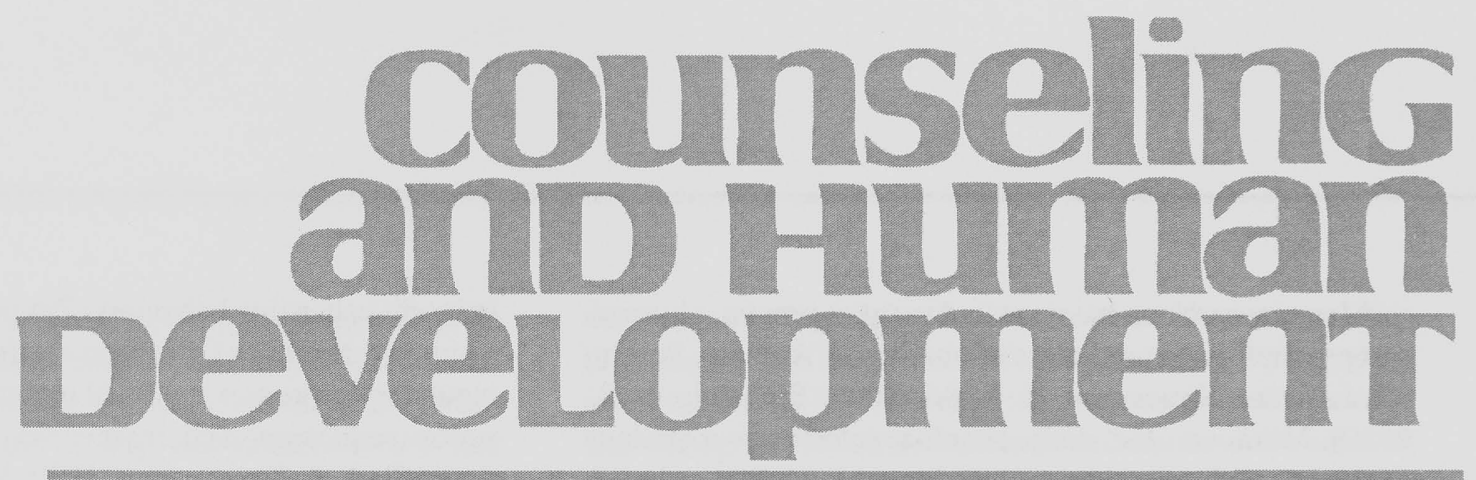

\title{
Acculturation and Self-Regulation as Determinants of Childhood Obesity
}

\author{
Regine Talleyrand and Anastasia Kitsantas
}

Within the past three decades childhood obesity rates have reached epidemic proportions (Ogden et al., 2006; Rocchini, 2002), signifying a serious public health issue. Specifically, in 2003-2004, approximately $17.1 \%$ of children and adolescents in the United States were overweight, which was a statistically significant increase from $13.9 \%$ in 1999-2000 (Ogden et al., 2006). Moreover, African American children and adolescents are nearly three times as likely to be overweight as their White counterparts (Centers for Disease Control and Prevention, 2002; Sealy, 2010) while Asian American and Hispanic adolescents born in the United States are more than twice as likely to be obese as their first-generation immigrant parents (Popkin \& Udry, 1998). The large number of racially and ethnically diverse children who are overweight or obese in comparison to their White counterparts suggests that racial and/or cultural factors may play a role in the prevalence of childhood obesity.

Obesity has several negative consequences in children including being a risk factor for various medical conditions such as heart disease, diabetes, and high blood pressure (Rocchini, 2002), as well as psychological distress including depression, low self-esteem, body dissatisfaction, and eating disturbances (Thompson \& Smolak, 2001). In fact, Horgen (2005) suggested that several medical conditions that were rarely seen in children are now becoming more common in obese children. Social and economic ramifications of obesity have also been demonstrated, including poorer academic achievement, lower educational attainment, inability to participate in the workforce, and social stigmatization (Clark, Slate, \& Viglietti, 2009; Gortmaker, Must, Perrin, Sotbol, \& Dietz, 1993; Krukowski et al., 2009; Sealy, 2010; Zametkin, Zoon, Klien, \& Munson, 2004). The fact that research has shown that obese children are likely to become obese adults (Daniels, 2006; Dietz, 1998) underscores the need to further examine the causal factors linked to obesity in youth - and to focus on factors that may be specific to racially and ethnically diverse children.

Dr. Talleyrand is an associate professor and coordinator of the Counseling and Development Program, and Dr. Kitsantas is a professor and coordinator of the Educational Psychology Program at George Mason University. 
Many researchers have studied various genetic, physical, and psychological predictors of obesity (D'Argenio, Pecchioli, Lorenzo, Siracusano, \& Troisi, 2009; Elovainio et al., 2010). Moreover, the study of self-regulation as a strategy for weight reduction has garnered much attention in health psychology research. Yet, the specific cultural factors that could potentially be related to childhood obesity remain insufficiently explored. Acculturation, a core concept in social and behavioral research, refers to the extent to which a child adopts the cultural norms of the dominant culture (e.g., White American culture) and enculturation refers to the extent to which a child retains his or her own indigenous cultural group norms (Berry, 2003; Kim \& Omizo, 2006). Very few studies have examined acculturation and enculturation as they relate to obesity, and their results have been contradictory. Nonetheless, the limited information available suggests that these cultural processes could be considered influential in the development of childhood obesity.

Self-regulation, in the context of health, refers to the degree to which an individual is able to proactively engage

\section{counseling and Human Development}

ISSN 0193-7375

COUNSELING AND HUMAN DEVELOPMENT (USPS 203-420)

is published monthly except June, July, and August as a service to counseling and human services professionals in schools, agencies, mental health centers, higher education, business and industry, and other private settings. This journal is available in microfilm from Serials Acquisitions, National Archive Publishing Company, P.O. Box 998, Ann Arbor, MI 48106-0998. Subscription rates: individual, $\$ 48$ per year; institutions, \$66 per year. Copyright (ㄷ 2010, Love Publishing Company. All rights reserved. Reproduction in whole or part without written permission is prohibited. Printed in the United States of America. Periodical postage is paid at Denver, Colorado. POSTMASTER: Send address changes to:

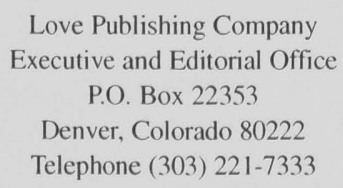

Mark Kiselica

The College of New Jersey
Paul Peluso Florida Atlantic University

Rita Chi-Ying Chung

George Mason University-Fairfax

Stanley F. Love Publisher
Carrie E. Watterson Senior Editor in health enhancing behaviors (Zimmerman, 1995). Although a number of studies have been conducted on the role of selfregulatory processes involved in initiating action, managing action sequences, and maintaining behavior change over time (Clark \& Zimmerman, 1990; Kitsantas, 2000), the relationships between acculturation and self-regulatory strategies on weight management remain unexplored. Given that large numbers of Black and Hispanic children are overweight and obese and the fact that self-regulation strategies have been employed in many weight reduction studies, it seems logical to explore the relationships among cultural factors, self-regulation strategies, and weight management (Bruss, Morris, \& Dannison, 2003; Javo, Ronning, \& Heyerdahl, 2004). Examining which cultural socialization experiences protect children from nondominant cultures from becoming overweight may aid us in preventing obesity among this population. Moreover, teaching children to selfregulate and internalize positive cultural experiences may further guarantee the effectiveness of health-promoting interventions.

The purpose of this article is to explore the interrelationships among acculturation processes, self-regulation, and obesity in youth. First, we will review research to determine whether the prevalence of childhood obesity is determined by variables such as race, ethnicity, and acculturation factors. Second, we will examine the relationship between selfregulation and weight management in youth. Third, we will propose a culturally relevant model that depicts self-regulation as a mediating factor between acculturation and obesity. Finally, we will present recommendations for counseling research, prevention, training, and practice.

\section{SOCIOECONOMIC STATUS, RACE, ETHNICITY, AND CHILDHOOD OBESITY}

Several causes have been attributed to the dramatic increase in percentages of overweight children, including lack of physical activity related to an increasingly sedentary lifestyle, unhealthy eating patterns, and genetic predispositions (Office of Surgeon General, 2001). Ten Eyck (2008) suggested that people commonly view obesity as a problem that occurs at the individual level, virtually ignoring contextual influences like the availability of healthy food choices, cultural norms regarding food and body appearance, and environmental contexts for physical activity. As a result, many individuals will perceive weight and obesity as issues that the individual solves him- or herself rather than examine cultural and societal influences that may also play a large role in an individual becoming overweight or obese. Yet, research suggests that a discussion of contextual factors (in particular, cultural contexts) is a necessity when studying obesity (Kumanyika, 2008). 
Researchers have reported several findings regarding the relationships among socioeconomic status (SES), race, ethnicity, and childhood obesity. Delva, Johnston, and O'Malley (2007) examined how obesity was related to SES and race in a sample of 8 th and 10 th grade students. Nationally representative data were collected from over 39,000 adolescent students. Analyses revealed that a male minority with low SES was more likely to have a body mass index (BMI) at or above the 85 th percentile. In terms of eating behaviors, healthy eating behaviors and healthy food consumption was negatively related to adolescents being at or above the 85 th percentile in terms of body weight, while frequency of watching TV was positively correlated. In fact, the authors suggested that these eating and TV-watching behaviors were more important in determining the weight of a child than family or parenting influences. Thus, these findings suggest that race, SES, unhealthy eating practices and physical behaviors (e.g., high levels of sedentary behaviors) in adolescents were linked to higher BMI more so than family or parenting influences.

Additionally, Delva, O'Malley, and Johnston (2006) examined the trends of overweight American students and how they could be differentiated by race/ethnicity and SES. Drawing from national data sets, a sample of over 100,000 middle and high school students were analyzed. Results revealed that minority, low SES, and higher grade level students were more likely to be overweight and have more unhealthy eating habits than White students in higher SES standing in lower grade levels. Moreover, the National Growth and Health Study (NGHS) conducted by Kimm et al. (1996) reported that for 9-10-year-old Black girls, risk for obesity did not vary across SES levels, whereas there was an inverse association for White girls. In general, parental education, not household income, exerted the greater effect on salutary behavioral factors, and the effect of parental education and SES was greater in White girls than in Black girls.

As a final point, evidence supporting the links among SES, race, and obesity rates can be derived from Richmond, Field, and Rich (2007), who examined how neighborhood characteristics were associated with the ethnic gap in adolescent inactivity. Using data from a national sample $(\mathrm{n}=$ 17,007), the results indicated that both Black and Hispanic adolescent boys and girls were more inactive than White boys and girls. In terms of neighborhood characteristics, the results suggested that both Hispanic and White girls engaged in the same level of inactivity when living in the same neighborhood, but Black girls and boys showed higher levels of inactivity regardless of the neighborhood. Adolescents' perceptions about the safety of their neighborhood were not associated with activity levels, but the rate of violent crime in the neighborhood was. Overall, the findings of these studies highlight that environmental characteristics (e.g., high crime neighborhood) and race/ethnicity may play a role in activity levels in children, and that the risks for obesity during childhood and adolescence can be differentiated by race, SES, and eating and physical activity behaviors. These findings also show that SES and parental education seem to be more of an obesity risk for White children than for children of color.

Certain cultural practices in the home have also been found to be related to obesity. For instance, Sen (2006) examined how adolescents' weight was related to how often their families sat down together for dinners and whether any differences correlated to ethnic background. Additionally Sen (2006) examined whether any longitudinal trends (1997 and 2000) could be discerned in that relationship. A total of 5,014 adolescents participated in their study. The results indicated that, in 1997, Whites' engagement in family dinners was related to a smaller likelihood of being overweight in 1997 and to an increased likelihood of no longer being overweight by 2000 . Therefore, for White adolescents, family unification during mealtimes was considered an important element in reducing overweight status. Interestingly enough, this relationship was not present for either Black or Hispanic adolescents. These differences can be explained by the fact that family unity and extended family involvement is a significant characteristic of Black and Latino cultures but may not play as large a role in the dominant White American culture (Comas-Diaz \& Greene, 1994; Helms \& Cook, 1999). Therefore, family involvement during mealtimes may not be an atypical concept for Black and Latino families. These findings also suggest that for Blacks and Hispanics, it may be more important to examine what is eaten rather than who is eating together.

Brewis (2003) also studied cultural aspects of obesity in a high SES sample of 219 young Mexican school children (ages 6-12) by assessing household, family, and parental variables; estimates of child health status; dietary and activity patterns; and demographic characteristics. Brewis (2003) found that permissive parenting styles and dietary intake did predict obesity in their sample. That is, because of a cultural belief that child fatness was a sign of good health and that food treats were a cultural index of parental caring, cultural feeding practices in the home environment explained the high rates of childhood obesity for this sample. Again, these results suggest that cultural attitudes and behaviors regarding food, eating, and body image may vary by race and ethnicity, which may also play into the high prevalence of obesity in racially and ethnically diverse children (Kumanyika, 2008).

In summary, it appears that the relationships among SES, race, and ethnicity have been examined in multiple ways and yielded significant results to support the impact of 
socio-cultural factors on the development of obesity. For instance, SES, race, and neighborhood characteristics tend to predict levels of physical activity, healthy eating behaviors, and overweight status in children. In fact, Kumanyika and Grier (2006) suggested that research typically shows that students and children from lower SES backgrounds and minority children tend to watch more television that exposes them more to the commercials that make high fat, low nutrition foods more desirable. Additionally, children and students of low income typically live in areas that have a high concentration of unhealthy fast food restaurants than in predominately White and higher class neighborhoods. These low-income areas may present additional barriers including unsafe and often dangerous streets and neighborhoods which provide inadequate areas for children to play and exercise. A review of this research also suggests that cultural practices and beliefs related to food behaviors, body appearance, and physical activity have also been related to overweight and obesity in youth. This potentially could imply that, for children of color, availability of foods; types of foods consumed; and cultural norms regarding food, appearance, and physical activity may be more important in determining levels of obesity than family involvement, parental education, and in some cases, SES. Next, we will review how acculturation processes have been examined in the obesity literature.

\section{ACCULTURATION PROCESSES AND OBESITY}

Culture refers broadly to the values, traditions, norms, behaviors, and rituals shared among groups of individuals (Helms \& Cook, 1999; Sue \& Sue, 2003; Triandis, 1995). Cultural values refers to learned beliefs, rituals, traditions, and other behaviors of a social group that are communicated from one generation to another and considered important, desirable, or attractive (Helms \& Cook, 1999; Kluckhohn \& Strodbeck, 1961; Sue \& Sue, 2003). That is, cultural values (1) guide an individual's behavior; (2) form the basis of group norms; (3) dictate the lifestyle of social groups; (4) provide group members with some degree of personal and social meaning; and (5) influence how individuals portray themselves, view the world, and interpret their experiences (Carter, 1991; Chung \& Bemak, 2002; Sue \& Sue, 2003; Triandis, 1995). Early conceptualizations of cultural values provided an initial framework for understanding cultural differences. However, most researchers in the United States who study cultural values have used Western-based approaches as the standard by which to assess cultural values and, as a result, have disregarded the unique and complex differences within and among various cultural groups (Carter, 1991; Constantine, Myers, Kindaichi, \& Moore,
2004; Yeh, Carter, \& Pieterse, 2004). Applying the construct of acculturation to counseling research models is one method of assessing differences within and among cultural groups.

The concept of acculturation also has had a long history in the social and behavioral sciences. Acculturation has often been viewed as the phenomenon that occurs when an individual comes into contact with more than one cultural system (Padilla, 1980). Thus, the acquisition of dominant cultural norms by members of a nondominant group is part of the acculturation process. This adaptation process may include but is not limited to adopting Western health behaviors, dietary intake, and home and family practices (e.g., languages spoken in the home) (Gordon-Larsen, Harris, Ward, $\&$ Popkin, 2003). However, some theorists argue that maintaining elements of one's traditional cultural values and norms versus adopting the cultural norms of the dominant culture can serve as a protective factor (Berry, 2003; Harris, 1998).

As previously described, acculturation is a process of adjusting one's own cultural values and belief systems to accommodate the norms and values of a different, more dominant group (Kim \& Abreu, 2001). Conversely, enculturation is a process through which an individual retains his or her own cultural values regardless of the dominant culture. For example, a Latino immigrant to the United States can be considered as acculturated if he or she adopts the values present in American society but enculturated if he or she retains the cultural norms from the country of origin. Additionally, Kim and Abreu (2001) suggest that acculturation falls along four different dimensions: cultural behaviors, values, knowledge, and identity. Enculturation can fall along these lines to create a continuum or a degree of enculturation or acculturation. In terms of where the individual falls along the lines of the different dimensions of acculturation, acculturation and enculturation can be further distinguished into different "statuses" (Berry \& Kim, 1988). Specifically, Berry and Kim (1988) suggested that, depending on the level of both enculturation and acculturation, an individual can experience 1) integration, 2) assimilation, 3) separation, and 4) marginalization. Integration represents an individual's level of balance between both acculturation and enculturation (e.g., biculturalism), while assimilation refers to individuals rejecting the norms of their indigenous culture while embracing the norms of the dominant culture (e.g., high levels of acculturation and low levels of enculturation). Separation represents the opposite of assimilation where one's own culture is retained while rejecting or becoming indifferent to the dominant culture (e.g., high levels of enculturation, low levels of acculturation) and finally, marginalization, where individuals reject the idea of both enculturation and acculturation. 
Acculturation has been associated with various mental and physical health outcomes including anxiety, low selfesteem, depression, and eating behaviors (Berry, 2003; Gold, 2007; Harris, 1998; Liou \& Bauer, 2007; Popkin \& Udry, 1998; Talleyrand, 1998, 1999). With respect to the obesity literature, only a few researchers have examined how the acculturation process affects the overweight status of immigrants as they acculturate to American society, and these results have been contradictory. In some cases, first-generation immigrants have better health despite lower SES based on which traditional cultural values and norms or maintained. Maintaining traditional cultural values and norms may explain childhood obesity rates among other nondominant cultural groups. That is, the reinforcement of indigenous ethnic group values can slow down the acculturation process and slow down rates of obesity, or it can contribute to obesity-promoting behaviors. Nonetheless, additional studies focused on identifying specific patterns of acculturation are important for delineating groups at risk for engaging in obesity-promoting behaviors. Also, identifying cultural norms and beliefs regarding food behaviors and body appearance in racially and ethnically diverse groups could contribute to our understanding of why the prevalence of obesity is higher in certain groups. Below we will review some of the studies that have attempted to isolate acculturation factors relevant to the development of obesity.

\section{Acculturation and Childhood Obesity Research}

In the obesity literature, several studies have specifically examined the link between acculturation and obesity in children. Gold (2007), based on her belief that secondgeneration immigrants tend to develop more diseases related to unhealthy eating or weight such as diabetes and high blood pressure, examined the food intake patterns of firstand second-generation immigrants. Specifically, in a sample of immigrant refugee parents from various countries and their adolescent children, Gold (2007) investigated how these immigrants coped with the differences in culture in terms of food. A series of 24 interviews suggested that, overall, the refugees had adapted their diets from their native country to the United States as a result of convenience and availability of ingredients. Although the second-generation adolescents in this study favored more American-type foods, their first generation parents did not and, as a result, consumed more traditional foods at home. That is, acculturation to dominant cultural food norms seemed to be more apparent in the second-generation immigrant participants.

Additionally, Liou and Bauer (2007) used a qualitative approach to examine the perceptions of Chinese Americans $(\mathrm{N}=40)$ related to obesity risk and prevention. The findings suggest that young Chinese Americans believed that the major factor related to significant weight gain was bad and unhealthy eating habits coupled with sedentary lifestyles. The participants also believed that major weight problems were rare in Asian populations while simultaneously reporting that they felt susceptible to obesity. Additionally, the participants thought that different external factors including media-generated advertisements and the convenience and popularity of fast foods impacted weight issues. Although the participants believed that consuming more traditional Chinese foods was more beneficial to health than American foods, acculturation factors influenced their increased consumption of less healthful American foods. In particular, since the participants were more acculturated to the dominant American culture than their older generation families, they were less likely to consume or prefer more healthful traditional Chinese foods to American foods.

Within the Latino community, acculturation processes have also impacted the health status of children. For instance, in a study focused on Hispanic participants, Buenrostro (2007) hypothesized that SES would moderate the relationship between acculturation and obesity risk in a sample of Hispanic participants $(\mathrm{N}=112)$. Results revealed that total caloric intake and Hispanic acculturation showed a quadratic relationship. Specifically, the relationship between caloric intake and acculturation was negative with individuals at lower BMIs, while this relationship became positive as individuals' BMI scores increased. In terms of moderation analyses, the results indicated that SES was a significant moderator of the relationship between protein intake and acculturation. These findings may lead to a greater understanding of the interaction among acculturation, socioeconomic status, and Hispanic obesity. Specifically, this study showed that the relationship among acculturation, SES, and obesity does not follow a linear pattern. The relationship between caloric intake and acculturation differs across the levels of obesity. Additionally, this pattern was found only in Hispanic populations; therefore, other racial/ethnic groups may show different relationships.

In another study targeting Hispanic youth, Gordon-Larsen et al. (2003) used a sample of 8,613 Hispanic youth (Mexican/Chicanos, Puerto Ricans, and Cubans) in grades 7-12 to explore mechanisms that explain the overweight differential across immigrant generations of Hispanic youth in the United States. Results showed that maintaining traditional values and norms (e.g., dietary intake, language isolation in the home) consistently explained the lower likelihood of being overweight among first generation Hispanic adolescents of all backgrounds. That is, foreign-born Hispanic American adolescents had healthier dietary patterns than their U.S.-born counterparts. Furthermore, inactivity increased with generation of U.S. residence, although the correlation was less striking than was the case with dietary patterns. Thus, without the beneficial pattern of enculturation factors 
such as indigenous diet and physical activity, first-generation Hispanic adolescents would have higher overweight prevalence. In summary, these results suggested that foreign-born Hispanic American adolescents maintaining their traditional cultural values and norms were less likely to be overweight. On the contrary, a rapid acculturation of a series of obesity-related behaviors that are allegedly typical of the dominant culture with first to subsequent generations of United States residents resulted in markedly higher rates of being overweight in second generation immigrants. These results also stress the need to examine within-ethnic group differences, since previous research has shown that Mexican children tended to be more overweight because their parents valued fatness as a sign of good health (Brewis, 2003).

Although African Americans may not be considered immigrants, African American cultural values are different from the dominant culture, thus African Americans also undergo acculturation and enculturation processes to some extent (Landrine, Richardson, Klonoff, \& Flay 1994). Thus, studying specific cultural factors in African Americans can also assist us in explaining why African Americans have the highest rates of obesity in the United States. In an effort to examine the influence of ethnicity and culture, Sealy (2010) conducted several focus groups to examine the perceptions, attitudes, food choices, and eating habits of African American, Caribbean Black, and Hispanic parents living in the United States and also explored what influenced these parents' food provisions for their children. Sealy (2010) found that two recurrent themes emerged from the data. One was the influence of ethnicity and culture on the foods selected and prepared for their children. That is, African American, Caribbean Black, and Hispanic parents tended to choose foods that were associated with their childhood eating practices, and some of these choices (e.g., consumption of fried foods and starches) were not necessarily healthy food choices. The second theme that emerged from the discussion of food choices was that of time constraints. Parents felt that their work schedules often limited their ability to prepare foods at home which also made fast food a more convenient option. Thus, it appears that, for some of these participants, maintaining traditional cultural values and time constraints had negative effects on food choices and preparation, which, in turn, could lead to obesity-promoting behaviors in their children.

A few researchers have explored the relationships among weight, SES, acculturation level, and perceptions of body image in ethnically diverse samples. For instance, Cachelin, Monreal, and Juarez (2006) surveyed Mexican American women's $(N=276)$ perceptions of attractiveness and found that more acculturated women preferred more thin body figures. However, they also found differences in the perceptions of obese (i.e., BMI > 30) and normal (i.e., BMI $=18.5-24.9$ ) weight women, where obese women held more positive perceptions than normal weight women of larger body silhouettes. The authors did not find any significant effects in that the BMI of women did not differ across levels of SES. The authors speculate that this may indicate that acculturation levels may be more of a determinant of body perceptions and a tendency to be overweight than SES levels. These results also suggest that the degree to which Mexican American women perceive thinner figures as being more attractive is related to acculturation level, because women who have lower levels of acculturation to the dominant culture do not perceive larger body figures as being overweight.

Similarly, Olvera, Suminski, and Power (2005) examined how BMI, gender, and acculturation impacted Latino mothers' $(\mathrm{N}=80)$ and children's views on attractiveness and body size. Most mothers were overweight, while approximately $30 \%$ of their children were overweight or at risk for being overweight. The findings revealed that children who were overweight or classified as at risk for being overweight had perceptions that thinner body images were more ideal than did children who were not overweight. In terms of gender differences, girls rated more obese figures more harshly in terms of attractiveness than boys. Acculturation was positively associated with the perception that thinner figures were more attractive. Interestingly, mothers' level of acculturation was positively related to girls' perception that thinner body images were ideal but was not related to boys' perceptions of body image.

Finally, Ball and Kenardy (2002) examined how ethnicity was related to different aspects of eating disorders in a diverse sample of Australian women. Specifically, different acculturation effects such as time since immigration were examined in relation to different levels of eating disorder behaviors. Ball and Kenardy (2002) hypothesized that women who had been in the country longer would show similar patterns of body weight, body weight dissatisfaction, and level of eating patterns/disorders to women who were Australian born. Additionally, the eating patterns and attitudes of ethnic minority women were compared to women native to Australia. Using a sample of 14,779 young women (ages 18-23), the results indicated that women from Englishspeaking countries other than Australia showed similar patterns of body dissatisfaction, dieting behaviors, and eating disorder behaviors. However, women born in non-Englishspeaking countries were less likely to show these at-risk behaviors for eating disorders. In terms of acculturation, the analysis revealed that acculturation was a significant predictor of women's BMI as well as body weight dissatisfaction, even when socioeconomic status was controlled for. Additionally, the longer the immigrant women had been in the country, the more similar their attitudes about their body and eating/dieting habits were to women who were Australian 
born. These results suggest that acculturation can impact food and eating behaviors as well as perceptions of body image and appearance.

Given that today's children are growing up in an increasingly diverse society and that higher rates of obesity are prevalent among racially and ethnically diverse children from nondominant cultures, we believe that it is imperative to study the link between acculturation and obesity-promoting behaviors. This review of empirical research suggests that cultural contexts impact ethnically diverse children's health behaviors and weight status. More specifically, length of stay in the United States and subsequent involvement with the dominant White American culture tended to play a role in weight gain in children. That is, in some racial and ethnic groups (i.e., Asian Americans), more exposure to the dominant White American culture resulted in adopting health-compromising eating behaviors that contributed to weight gain. In other cases, children who internalize their parent's traditional cultural attitudes regarding health and food can be negatively at risk for becoming overweight. This appears to depend on the cultural group's perceptions regarding food behaviors, food preparation, and body appearance. For example, it appears that traditional cultural norms regarding heavier body ideals are evident in African American and Latino cultures, which can have a positive impact on how these groups perceive being overweight regardless of the medical ramifications. Nonetheless, people can engage in certain strategies to regulate what cultural attitudes and practices to adopt while engaging in strategies to regulate their health behaviors. We will now turn to reviewing the relationship between selfregulation and weight behaviors in children.

\section{THE ROLE IN SELF-REGULATION IN PROMOTING WEIGHT MAINTENANCE IN CHILDHOOD OBESITY}

From a social-cognitive perspective, self-regulation of health refers to self-generated thoughts, feelings, and actions that are planned and initiated towards the attainment of personal goals (Zimmerman, 1995). Self-regulated learners engage in a number of key self-regulatory processes or strategies including setting goals (specifying intended actions or outcomes), using a variety of task strategies (advantageous methods for maintain health-enhancing behaviors), self-monitoring (observing and tracking performance and outcomes), and evaluating their progress systematically to attain their goals. Engaging in these self-regulatory strategies enhances students' motivation to engage in healthenhancing behaviors and promotes successful weight maintenance (Israel, Guile, Baker, \& Silverman, 1994; Kitsantas, 2000; Macdonald \& Palfai, 2008; Wing, Tate, Gorin, Raynor, \& Fava, 2006).
According to Clark and Zimmerman's (1990) triadic model of health regulation, individuals act (behavior) within a given context (environment) and react to the outcomes (personal) of the behavior. These three dimensions of selfregulation (behavior, environment, and personal) are not independent; instead, they are interactive in nature. Successful self-regulation of the three dimensions involves three constructs: commitment, self-efficacy, and strategies (Clark \& Zimmerman, 1990). For example, an individual who sets a goal of losing 10 pounds in a month must engage in behaviors and strategies (e.g., buying healthy, low calorie foods and rewarding him- or herself with a treat only once a week) that would facilitate weight loss. However, in order for individuals to choose to engage in those behaviors, they must be genuinely committed to losing those 10 pounds. In other words, individuals must be committed to their goals before they are able to deliberately and successfully engage in strategies to regulate their behavior towards goal attainment. Self-efficacy (the degree to which one feels capable in performing a task under specific conditions) is also a critical variable that contributes to individuals' success at regulating their health, given the fact that they need to self-initiate and direct their own actions towards achieving a certain goal without the assistance or reliance on others. Therefore, selfefficacy may serve as a determinant of successful engagement in self-regulated learning (Bandura, 1986; Zimmerman, 2008).

Based on the triadic model of self-regulation, the home and family environments are critical to the acquisition of knowledge and the development of self-regulatory skills and self-efficacy beliefs (Bandura, 1986; Rosenthal \& Zimmerman, 1978). By social means such as modeling various health strategies, including goal setting and self-monitoring, descriptions, and written instructions, parents are able to convey important information to their children about health and disease. Children constantly emulate their parent's strategies about health and ask questions to achieve control of their own health functioning. For example, Arredondo et al. (2006) examined how parenting strategies impacted children's eating habits and health behaviors with 812 Latino parents and their children enrolled in kindergarten through second grade. The results showed that parents' use of positive reinforcement and monitoring strategies were positively related to their children's eating habits and health behaviors. Therefore, home practices can foster or undermine the development of good health behaviors in children when they internalize parental attitudes, self-efficacy beliefs, and standards (Bandura, 1986; Zimmerman, 1999).

Numerous research studies have documented the important role that self-regulatory strategies and self-efficacy beliefs play on children's and adolescents' ability to maintain 
their weight (Braet \& Van Winckel, 2000; Gamble, Parra, \& Beech, 2009; Israel et al., 1994; Macdonald \& Palfai, 2008; Riggs, Sakuma, \& Pentz, 2007; Wing et al., 2006). In fact, Wing et al. (2006) suggested that it is important for obesity and weight maintenance programs to incorporate self-regulation because of the common relapses that individuals who successfully lose weight experience. In fact, simply designing a program to help individuals lose weight is straightforward, but designing a program that is effective in maintaining weight loss is the challenge. Wing et al. (2006) addressed this issue specifically through a self-regulatory weight management program designed to address both weight loss and weight maintenance. The program was designed with self-regulatory components such as goal setting, self-monitoring, eating behaviors, and strategies where participants were trained to monitor their weight in relation to the goal weight and, depending on the difference, adjust their weight loss strategies and eating behaviors to facilitate goal attainment. This intervention was delivered both through online and face-to-face sessions. A control group received just quarterly newsletters. The results revealed that, compared to the control group, both participants in the online and face-to-face groups experienced a decreased risk of regaining weight. However, participants in the face-toface intervention session group were the ones who maintained the lowest amount of regained weight.

In another experimental study, Israel et al. (1994) investigated the impact of a self-regulation intervention with 26 overweight children and adolescents and at least one of their parents. Two treatment groups were formed: one group with only a standard intervention, where parents were given a set of rules and procedures to follow, and an enhanced involvement condition, where the same intervention from the standard treatment group was provided in addition to training in child self-regulation. Although both groups experienced weight loss during the 6-month treatment period, the experimental group participants experienced additional long-term benefits in the follow-up results.

Researchers who have also focused on studies involving ethnic minorities have found similar findings. Riggs et al. (2007) examined how an intervention targeted on reducing the risk of obesity during childhood impacted Latino and Asian American children's attitudes towards food as well as physical activity. Specifically, Riggs et al. (2007) attempted to equip students with more impulse control, effective decision-making skills, and social competence to combat childhood obesity. Following the intervention, the results revealed that students who were more self-regulated in their dietary intake and impulse control were associated with more healthful food choices and more adaptive TV viewing behaviors.

Other studies have also focused on the role of motivational variables on weight maintenance (Gamble et al.,
2009; Wing et al., 2006). For example, Gamble et al. (2009) examined how different variables such as physical activity, shared family activities, and self-efficacy for healthy eating interacted with or affected obesity in adolescent children. The level of obesity was measured by the BMI. A total of 168 ethnically diverse adolescent boys and girls participated in the study. The results indicated that although none of the variables contributed independently to BMI, they did show interactive patterns. Specifically, out of all the three independent variables examined, self-efficacy to healthy eating was found to be the only significant moderator of the relationship between the level of physical activity and BMI percentile, where adolescents who more frequently engaged in physical activities along with higher levels of self-efficacy for healthy eating had lower BMI percentiles than adolescents who were less frequently engaged in physical activities and had lower levels of self-efficacy for healthy eating. The authors suggested that this finding shows that researchers must examine the interactive nature of different weight loss behaviors and cognitions instead of independent effects to capture the relationships among these variables accurately.

Additionally, Macdonald and Palfai (2008) examined how goals and self-regulation can predict the exercise habits of university women longitudinally. Specifically, the authors hypothesized that goal-related appraisal, self-regulation, and affect would be significant predictors of future fitness behaviors even with BMI being controlled. A total of 97 women participated in the study. Fitness behaviors were measured with exercise calendars in which the participants self-reported the type of exercise in which they engaged as well as the frequency and duration of the exercise. Hierarchical regression analyses controlling for baseline measures of exercise behaviors and BMI revealed that approximately $60 \%$ of the variation in future exercise behaviors was explained by the different regulatory and motivational goal factors. Specifically, these findings suggested that having strong self-regulation skills and motivational beliefs are important in the maintenance of exercise behaviors.

Self-regulatory skills and motivational constructs have also been embedded in other training programs (Jelalian \& Mehlenbeck, 2002). Jelalian and Mehlenbeck (2002) evaluated the effectiveness of a Peer-Based Skills Training (PBST) component to supplement a 16-week traditional cognitive-behavioral weight management intervention. Participants included 16 adolescents (13-16 years). The supplemental PBST course included group activities of mental and physical natures designed to promote development of trust, social skills, and self-confidence. Results of this study support the intervention in that the adolescents lost weight and these losses also generated greater self-confidence in appearance and physical self-worth. 
Racial differences in motivational variables have also been examined in the self-regulation literature, but to a far lesser extent. Powell (2007) studied racial differences between African American and White students in terms of the relationship between body weight and different psychological factors such as self-esteem, satisfaction, and selfperception in a sample of 160 college students. The results revealed that males had higher BMI scores than females and African American students had higher BMI scores than White students. Additionally, BMI was negatively related to factors such as self-esteem and self-perceptions such that participants with higher levels of BMI had less self-esteem and self-perceptions than participants with lower BMI levels. Moreover, the students who were either under or at normal weight had more adaptive levels of self-esteem and selfperceptions than obese students. Finally, females and African American students had more adaptive levels of selfesteem than male and White students. These results suggest that self-esteem and BMI may not be inversely correlated in African American students, potentially because of larger body ideals in the African American community (Powell, 2007).

Overall, the research summarized above shows that selfregulation and motivation processes can help adolescents and children maintain or develop a healthy weight. Specifically, processes such as monitoring (Arredondo et al. 2006), weight control strategies (Blow, Taylor, Cooper, \& Redfearn, 2009), self-efficacy (Gamble et al., 2009; Jelalian \& Mehlenbeck, 2002; Powell, 2007), and goals (Macdonald \& Palfai, 2008) are important factors in determining peoples' weight loss behaviors and successful weight maintenance. However, as we have discussed in this article, it is important to note that culture also influences the internalization, implementation, and maintenance of weight self-regulation strategies and motivational beliefs. Interestingly enough, researchers studying self-regulation have not examined extensively various socio-cultural factors and their relationship to self-regulation. In fact, several studies we reviewed were lacking racial/ethnic demographic data when describing their participants. We, however, maintain that cultural variables can contribute to our understanding of weight management in children. Next, we will present our proposed model of childhood obesity by examining the relationships among acculturation, self-regulation, and weight management.

\section{ACCULTURATION AND SELF-REGULATION AS DETERMINANTS OF CHILDHOOD OBESITY: A PROPOSED MODEL}

Our childhood obesity model is guided by Social Cognitive Theory (SCT), which postulates that multiple psychosocial factors including socio-cultural influences (e.g. level of acculturation and enculturation, SES, cultural food practices and beliefs) impact a child's weight status. Moreover, we contend that self-regulation practices and selfbeliefs can also mediate the relationship between the sociocultural factors and children's health outcomes (Bandura, 1986; Bruss et al., 2003; Clark \& Zimmerman, 1990; Gamble et al., 2009; Gortmaker et al., 1993; Riggs et al., 2007). Generally, in health-related research, researchers have studied each of these constructs (i.e., acculturation, family practices, and self-regulation of health) independently rather than evaluating them collectively despite the fact that contextual factors may indeed influence the extent to which individuals engage in self-regulation (Bruss et al., 2003; Javo et al., 2004). We believe that, given the increasingly diverse student population and increasing childhood obesity rates, our model provides an innovative, culturally-based self-regulation model for addressing health concerns among racially and ethnically diverse children and their parents (see Figure 1). It is hypothesized that contextual factors, which include 1) SES, race, and gender; 2) family cultural practices; and 3) level of acculturation, impacts children's ability to maintain their weight through the use of self-regulatory strategies and self-efficacy to control eating habits. Additionally, it is hypothesized that self-regulation strategies and self-efficacy to control eating habits are interdependent. That is, children may be knowledgeable regarding strategies they can use to regulate their weight, but if they do not feel self-efficacious in using these strategies, they will be less likely to implement them when necessary.

Ultimately, the relationship between self-regulatory processes and eating habits/weight management may depend on the culture of the individual. As previously discussed, the values of a specific culture may be a direct factor in weight gain, like in Latino and African cultures where being overweight has been historically associated with health and wealth (Brewis, 2003; Villarosa, 1994), while in Asian cultures, enculturation is associated with more healthful eating habits, as opposed to acculturating to dominant White American eating habits (Liou \& Bauer, 2007).

Although acculturation is a term specifically used to describe the cultural assimilation process of ethnic minorities who have immigrated to another country, this term can also apply to minority groups who are already established in the United States. For example, although African Americans are part of the American culture, they are also considered a subculture that has different indigenous cultural values and lifestyles than White Americans (Kimm et al., 1996; Kumanyika, 2008; Sen, 2006), which may translate into differences in food consumption, body image, as well as actual weight. Teaching children to self-regulate their weight is closely linked to these processes (depending on whether acculturation or enculturation should be implemented). 
Contextual

Influences

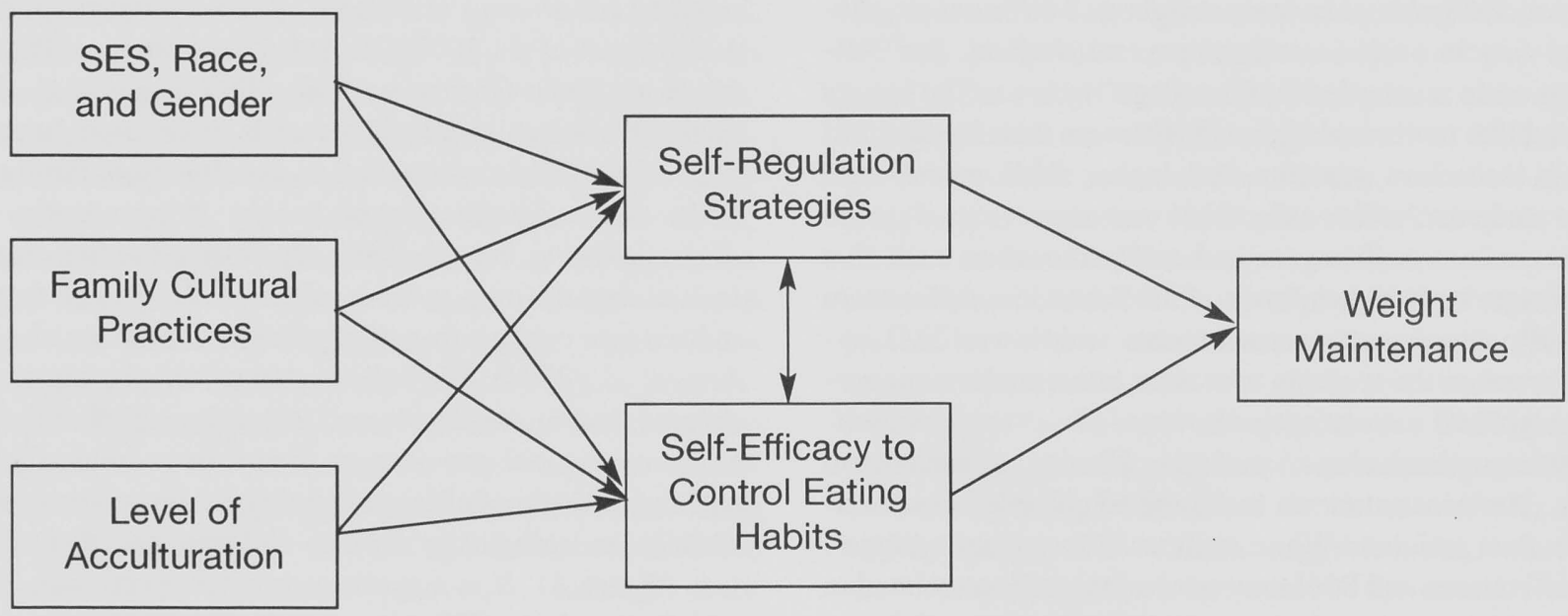

FIGURE 1

Hypothetical Model Examining the Relationships Among Acculturation, Self-Regulated Strategies, and Self-Efficacy and Weight Maintenance

Family and schools could play an important role in teaching children a variety of self-regulatory strategies and boosting their self-efficacy beliefs to use these strategies to manage their weight.

\section{FUTURE DIRECTIONS FOR THE FIELDS OF COUNSELING AND PSYCHOLOGY}

Despite a growing body of empirical evidence supporting relationships among race, culture, and obesity in youth (Thompson \& Smolak, 2001), neither a theoretical framework nor empirical literature currently exists in which ethnically diverse children's cultural socialization experiences and obesity are linked. Further, the relationships between acculturation and self-regulation strategies on weight management remain unexplored. Given that the increasing rate of obesity is expensive and is taking a strong financial toll on both the general population and the government (Foerster et al., 2007), research in early childhood obesity can inform obesity prevention programs and reduce the financial stress of addressing health issues. We have attempted to propose a model that identifies cultural socialization and self-regulation factors as socio-cultural determinants of childhood obesity. Some researchers contend that, in terms of development, early childhood is a stage where healthy habits can be emphasized and turned into long-term healthy habits (Foerster et al., 2007). However, in order to do so, more research needs to be done in order to understand how obesity is caused and how interventions can be differentiated to address the different causes.

\section{Implications for Counseling Research, Practice, and Training}

We believe that our model can advance the obesity literature in several ways. First, identifying which specific elements of the acculturation process (e.g., cultural norms regarding food and physical appearance) contribute to childhood obesity can be very helpful in understanding the prevalence of obesity among ethnically diverse children. That is, developing and using culturally appropriate assessment tools to measure the cultural and racial socialization experiences of children of color may provide more consistent results to aid us in understanding specific factors related to childhood obesity. It is evident from the limited number of studies that did include acculturation as a construct of interest that the "measure" of acculturation was inconsistent. Some studies measured acculturation by assessing participants' perceptions of body image and size (Cachelin et al., 2006; Olvera et al., 2005), while others used generational status (Liou \& Bauer, 2007), native language use (GordonLarsen et al, 2003; Sussner, 2007), and types of food consumed (Buenestro, 2007; Gold, 2007; Gordon-Larsen et al., 2003) as indicators of levels of acculturation and enculturation. Further, exploring the role of self-regulation in this 
model can also be useful in building program effectiveness. Specifically, if we follow Clark and Zimmerman's (1990) perspective on self-regulated learning about health, we can systematically design research studies that take into account constructs and factors such as acculturation, SES, and selfregulatory strategies training. If these constructs are taken into consideration, we are likely to not only provide people with knowledge regarding a healthy lifestyle, we can also equip them with the proper strategies to actually engage in healthy behaviors and the motivation to commit to a different, more healthy eating and exercise routine. Finally, we suggest that qualitative and quantitative research methods should be used to potentially expand our understanding of the role of each construct. Given the lack of attention given to these constructs, using interviews and focus groups with racially and ethnically diverse participants would allow us to gather themes and constructs of interest. We could then follow up with quantitative studies to measure whether or not socio-cultural factors are related to the weight status and management of ethnically diverse youth.

Testing a model of this sort can also offer counselors new methods of culturally relevant assessment, intervention, and training. Assessing acculturation levels when working with clients can give counselors additional insight as to which cultural values are most salient for their clients and how these values impact clients' health and eating attitudes and behaviors. Counselors can also provide more accurate diagnoses and treatment plans with a better understanding of predictive factors contributing to obesity in children of color. In addition, counselor training programs, accreditation standards, and national organizations can continue to emphasize the necessity for culturally competent clinicians who can recognize and understand acculturative processes in children rather than use ethnocentric approaches in diagnosing and treating clients.

\section{Implications for Obesity Prevention Programs}

The development of culturally sensitive childhood obesity prevention programs in schools and communities could help with the increasingly growing problem of childhood obesity. Since obese children are likely to become obese adults (Daniels, 2006; Dietz, 1998; Thompson \& Smolak, 2001), it is imperative to break the cycle at an earlier age. In the last several years, numerous obesity prevention programs targeting ethnically diverse children have been implemented and evaluated. For example, Hernandez (2008) examined how a culturally relevant community intervention program designed specifically to target increasing the healthy behaviors of overweight Latino children as well as preventing obesity within the Latino community was successful or unsuccessful. After completion of nutritional education, behavioral health, and physical activity programs, individuals who had participated in the program had significantly lowered their BMI percentile and held better approaches to nutrition, physical activity, and self-esteem. However, individuals who did not successfully complete the program expressed difficulties such as diabetes in the family and different contextual challenges. Additionally, Stolley and Fitzgibbon (1997) examined the effectiveness of a culturally relevant obesity prevention program among lower income, inner city preadolescent African American girls and their mothers during a 12 -week treatment program that included grocery shopping at local supermarkets, incorporating culturally relevant music for increasing physical activity, and dissecting high-fat recipes and showing changes. Results from this study indicated that the mothers in the treatment group showed significant differences in fat intake and percentage of calories from fat, while their daughters showed some improvement, too, but changes were not significant in this group of participants. Finally, the Girls Health Enrichment Multi-Site Studies (GEMS) consisted of a multicenter research program targeted at preventing excessive weight gain in low-income African American girls (Story et al., 2003). These researchers developed several after-school short-term intervention programs for African American girls focusing on increasing physical activity and healthy eating through the use of recruitment strategies and intervention strategies (e.g., dancing, family and community involvement) that were consistent with African American cultural values, traditions, and customs. Results from these interventions suggested that the most effective intervention strategies included after-school dance classes, a family-based intervention to reduce television viewing, and psycho-educational materials targeting the development of healthy eating and activity skills for youth. These intervention strategies did not focus on obesity prevention but rather on a holistic concept of health that included high self-esteem, spirituality, and cultural awareness, as well as physical health.

All of these obesity prevention programs have targeted racially and ethnically diverse children in an effort to reduce obesity rates among this population. Results from these research studies have demonstrated that incorporating culturally relevant elements into these program designs improves the retention and success rates. This suggests that prevention programs need to address how to most effectively bring about health changes while simultaneously taking into account the values of that culture.

\section{SUMMARY}

In this article, we have reviewed the study of culture and self-regulation in relation to childhood obesity. This review has suggested that several factors are important in 
understanding why ethnically diverse children have higher rates of obesity in comparison to their White counterparts. Factors such as acculturation and enculturation levels seem to play a role in children of color's food intake, activity levels, and perceptions of body image. The research also suggests that levels of acculturation and enculturation can contribute to obesity-promoting or obesity-reducing behaviors, based on the indigenous cultural norms and practices of the racial or ethnic group. Additional factors such as SES and environmental contexts also seem to affect obesity-promoting behaviors, since individuals from lower SES families and from low-income neighborhoods may have less access to healthy food choices and adequate play space; however, SES seems to be more of a significant factor for White children than for other racial and ethnic groups. The relationship between self-regulation and weight management may also be different between racial and ethnic groups based again on cultural norms regarding food and body image; however, to our knowledge this relationship has not been tested. Additionally, further questions remain. Are family cultural practices significantly linked to how individuals manage their weight? Does SES and acculturation add to the variance we see in weight status? How do self-regulation and self-efficacy mediate this relationship? By addressing these cultural contexts we could potentially add to researchers' and counselors' understanding of why ethnically diverse children experience higher incidences of overweight and obesity and also help us develop obesity prevention programs that incorporate these elements.

\section{REFERENCES}

Arredondo, E., Elder, J., Ayala, G., Campbell, N., Baquero, B., \& Duerksen, S. (2006). Is parenting style related to children's healthy eating and physical activity in Latino families? Health Education Research, 21(6), 862-871.

Ball, K., \& Kenardy, J. (2002). Body weight, body image, and eating behaviours: Relationships with ethnicity and acculturation in a community sample of young Australian women. Eating Behaviors, 3(3), 205-216.

Bandura, A. (1986). Social foundations of thought and action. Englewood Cliffs, NJ: Prentice-Hall.

Berry, J. W. (2003). Conceptual approaches to acculturation. In K. M. Chun, P. B. Organista, \& G. Marin, (Eds.), Acculturation: Advances in theory, measurement, and applied research (pp. 17-38). American Psychological Association: Washington, DC.

Berry, J. W., \& Kim, U. (1988). Acculturation and mental health. In P. R. Dasen, J. W. Berry, \& N. Sartorius (Eds.), Health and cross-cultural psychology: Toward applications (pp. 207-236). Newbury Park, CA: Sage.

Blow, J., Taylor, T., Cooper, T., \& Redfearn, C. (2009). Correlates of weight concern and control in a Hispanic college student sample. Eating Behaviors, 11, 6-10.

Braet, C., \& Van Winckel, M. (2000). Long-term follow-up of cognitive behavioral treatment program for obese children. Behavior Therapy, 3l(1), 55-74.
Brewis, A. (2003). Biocultural aspects of obesity in young Mexican schoolchildren. American Journal of Human Biology, 15, 446-460.

Bruss, M. B., Morris, J., \& Dannison, L. (2003). Prevention of childhood obesity: Sociocultural and familial factors. Journal of the American Dietetic Association, 103(8), 1042-1045.

Buenrostro, L. (2007). Acculturation and obesity among Latinos: Reconciling inconsistent findings. (Doctoral dissertation, Rosalind Franklin University of Medicine and Science, 2006). Dissertation Abstracts International, 67, Retrieved from PsycINFO database.

Cachelin, F., Monreal, T., \& Juarez, L. (2006). Body image and size perceptions of Mexican American women. Body Image, 3(1), $67-75$.

Carter, R. T. (1991). Cultural values: A review of empirical research and implications for counseling. Journal of Counseling and Development, 70(1), 164-173.

Centers for Disease Control and Prevention, National Center for Health Statistics. (2002). Obesity still on the rise, new data show. Retrieved from http://www.cdc.gov/nchs/releases/02news/obesityonrise.htm

Chung, R., \& Bemak, F. (2002). The relationship between culture and empathy in cross-cultural counseling. Journal of Counseling and Development, 80, 154-159.

Chung, R., Bemak, F., Ortiz, D., \& Sandoval-Perez, P. (2008). Promoting the mental health of immigrants: A multicultural/social justice perspective. Journal of Counseling \& Development, 86(3), 310-317.

Clark, D., Slate, J., \& Viglietti, G. (2009). Children's weight and academic performance in elementary school: Cause for concern? Analyses of Social Issues and Public Policy (ASAP), 9(1), 185-204.

Clark, N. M., \& Zimmerman, B. J. (1990). A social cognitive view of self-regulated learning about health. Health Education Research, 5(3), 371-379.

Comas-Díaz, L., \& Greene, B. (1994). Women of color: Integrating ethnic and gender identities in psychotherapy. New York: Guilford Press.

Constantine, M., Myers, L., Kindaichi, M., \& Moore, J. (2004). Exploring indigenous mental health practices: The roles of healers and helpers in promoting well-being in people of color. Counseling and Values, 48(2), 110-125.

D’Argenio, A., Mazzi, C., Pecchioli, L., Di Lorenzo, G., Siracusano, A., \& Troisi, A. (2009). Early trauma and adult obesity: Is psychological dysfunction the mediating mechanism? Physiology \& Behavior, 98(5), 543-546. doi:10.1016/j.physbeh.2009.08.010

Daniels, S. (2006). The consequences of childhood overweight and obesity. The Future of Children, 16(1), 47-67. doi:10.1353/foc. 2006.0004

Delva, J., Johnston, L., \& O’Malley, P. (2007). The epidemiology of overweight and related lifestyle behaviors: Racial/ethnic and socioeconomic status differences among American youth. American Journal of Preventive Medicine, 33(4), 178-186.

Delva, J., O'Malley, P., \& Johnston, L. (2006). Racial/ethnic and socioeconomic status differences in overweight and health-related behaviors among American students: national trends 1986-2003. Journal of Adolescent Health, 39(4), 536-545.

Dietz, W. (1998). Health consequences of obesity in youth: Childhood predictors of adult disease. Pediatrics, 101(3), 518. Retrieved from Academic Search Complete database.

Elovainio, M., Pulkki-Råback, L., Kivimäki, M., Jokela, M., Viikari, J., Raitakari, O., et al. (2010). Lipid trajectories as predictors of depressive symptoms: The Young Finns Study. Health Psychology, 29(3), 237-245. doi:10.1037/a0018875. 
Foerster, S., Silver, L., Kohatsu, N., Frieden, T., Bassett, M., \& Horton, M. (2007). Childhood obesity on the front lines. American Journal of Preventive Medicine, 33(4), 175-177.

Gamble, H., Parra, G., \& Beech, B. (2009). Moderators of physical activity and obesity during adolescence. Eating Behaviors, 10, 232-236.

Gold, A. (2007). Changing foodways: Generational communication in a new American/refugee population. (Doctoral dissertation, North Dakota State University, 2007). Dissertation Abstracts International, 68, Retrieved from PsycINFO database.

Gordon-Larsen, P., Harris, K. M., Ward, D. S., \& Popkin, B. M. (2003). Acculturation and overweight-related behaviors among Hispanic immigrants to the U.S.: The National Longitudinal Study of Adolescent Health. Social Science \& Medicine, 57, 2023-2034.

Gortmaker, S. L., Must, A., Perrin, J. M., Sobol, A. M., \& Dietz, W. H. (1993). Social and economic consequences of overweight in adolescence and young adulthood. The New England Journal of Medicine, 329(14), 1008-1012.

Hafetz, J. (2007). The intergenerational transmission of health knowledge and behaviors: An evaluation of the Go!Kids obesity prevention program. (Doctoral dissertation, Fordham University, 2007). Dissertation Abstracts International, 68, Retrieved from PsycINFO database.

Harris, K. M. (1998). The health status and risk behavior of adolescents in immigrant families. In D. Hernandez (Ed.), Children of immigrants: Health, adjustment, and public assistance (pp. 286-347). Washington, DC: National Academy Press.

Helms, J., \& Cook, D. (1999). Using race and culture in counseling and psychotherapy: Theory and process. Needham Heights, MA: Allyn \& Bacon.

Hernandez, E. A. (2008). A validation study on a Latino childhood overweight program. (Doctoral dissertation, Alliant International University, 2008). Dissertation Abstracts International, 68, Retrieved from PsycINFO database.

Horgen, K. (2005). Big food, big money, big children. Childhood lost: How American culture is failing our kids (pp. 123-135). Westport, CT: Praeger/Greenwood.

Israel, A. C., Guile, C. A., Baker, J. E., \& Silverman, W. K. (1994). An evaluation of enhanced self-regulation training in the treatment of childhood obesity. Journal of Pediatric Psychology, 19(6), 737-749.

Javo, C., Ronning, J., \& Heyerdahl, S. (2004). Child-rearing in an indigenous Sami population in Norway: A cross-cultural comparison of parental attitudes and expectations. Scandinavian Journal of Psychology, 45, 67-78.

Jelalian, E., \& Mehlenbeck, R. (2002). Peer-enhanced weight management for overweight adolescents: Some preliminary findings. Journal of Clinical Psychology in Medical Settings, 9(1), 15-23.

Kim, B. S. K., \& Abreu, J. M. (2001). Acculturation measurement: Theory, current instruments, and future directions. In J. G. Ponterotto, J. M. Casas, L. A. Suzuki, \& C. M. Alexander (Eds.), Handbook of multicultural counseling (2nd ed., pp. 394-424). Thousand Oaks, CA: Sage.

Kim, B. S. K., \& Omizo, M. M. (2006). Behavioral acculturation and enculturation and psychological functioning among Asian American college students. Cultural Diversity and Ethnic Minority Psychology, 12, 245-258.

Kimm, S. Y., Obarzanek, E., Barton B. A., Aston, C. E., Similo, S. L., Morrison, J. A. ... McMahon, R. P. (1996). Race, socioeconomic status, and obesity in 9- to 10-year-old girls: The NHLBI Growth and Health Study. Annals of Epidemiology, 6(4), 266-275.

Kitsantas, A. (2000). The role of self-regulation strategies and self-efficacy perceptions in successful weight loss maintenance. Psychology \& Health: An International Journal, 15, 811-820.
Kluckhohn, F. R. \& Strodtbeck, F. L. (1961). Variations in value orientations. Evanston, IL: Row, Peterson.

Krukowski, R., West, D., Perez, A., Bursac, Z., Phillips, M., \& Raczynski, J. (2009). Overweight children, weight-based teasing and academic performance. International Journal of Pediatric Obesity, 4(4), 274-280.

Kumanyika, S. (2008). Ethnic minorities and weight control research priorities: Where are we now and where do we need to be? Preventive Medicine: An International Journal Devoted to Practice and Theory, 47(6), 583-586.

Kumanyika, S., \& Grier, S. (2006). Targeting interventions for ethnic minority and low-income populations. The Future of Children, 16(1), 187-207.

Landrine, H., Richardson, J., Klonoff, E., \& Flay, B. (1994). Cultural diversity in the predictors of adolescent cigarette smoking: The relative influence of peers. Journal of Behavioral Medicine, 17(3), 331-346.

Liou, D., \& Bauer, K. (2007). Exploratory investigation of obesity risk and prevention in Chinese Americans. Journal of Nutrition Education and Behavior, 39(3), 134-141.

Macdonald, A., \& Palfai, T. (2008). Predictors of exercise behavior among university student women: Utility of a goal-systems/selfregulation theory framework. Personality and Individual Differences, 44(4), 921-931.

Office of the Surgeon General, U.S. Department of Health and Human Services. (2001). The Surgeon General's call to action to prevent and decrease overweight and obesity. Rockville, MD: Public Health Service, Office of the Surgeon General.

Ogden, C., Carroll, M., Curtin, L., McDowell, M., Tabak, C., \& Flegal, K. (2006). Prevalence of overweight and obesity in the united states, 1999-2004. JAMA: Journal of the American Medical Association, 295(13), 1549-1555.

Olvera, N., Suminski, R., \& Power, T. (2005). Intergenerational perceptions of body image in Hispanics: Role of BMI, gender, and acculturation. Obesity Research, 13(11), 1970-1979.

Padilla, A. (Ed.). (1980). Acculturation: Theory, models, and some new findings. Boulder, CO: Westview.

Popkin, B. M., \& Udry, J. R. (1998). Adolescent obesity increases significantly in second- and third-generation U.S. immigrants: the National Longitudinal Study of Adolescent Health. Journal of Nutrition, 128, 701-706.

Powell, T. (2007). The impact of obesity on well-being among AfricanAmerican and Caucasian college students. (Doctoral dissertation, Howard University, 2007). Dissertation Abstracts International, 67, Retrieved from PsycINFO database.

Richmond, T., Field, A., \& Rich, M. (2007). Can neighborhoods explain racial/ethnic differences in adolescent inactivity? International Journal of Pediatric Obesity, 2(4), 202-210.

Riggs, N., Sakuma, K., \& Pentz, M. (2007). Preventing risk for obesity by promoting self-regulation and decision-making skills: Pilot results from the PATHWAYS to health program (PATHWAYS). Evaluation Review, 31(3), 287-310.

Rocchini, A. P. (2002). Raising heart healthy children. Pediatrics International, 4l(6), 597-602.

Rosenthal, T. L., \& Zimmerman, B. J. (1978). Social learning and cognition. New York: Academic Press.

Sealy, Y. (2010). Parents' food choices: Obesity among minority parents and children. Journal of Community Health Nursing, 27(1), 1-11.

Sen, B. (2006). Frequency of family dinner and adolescent body weight status: Evidence from the National Longitudinal Survey of Youth, 1997. Obesity, 14(12), 2266-2276.

Stolley, M., \& Fitzgibbon, M. (1997). Effects of an obesity prevention program on the eating behavior of African American mothers and 
daughters. Health Education \& Behavior, 24(2), 152-164. doi:10.1177/109019819702400204

Story, M., Sherwood, N. E., Himes, J. H., Davis, M., Jacobs, D. R., Cartwright, Y., ... Rochon, J. (2003). An after-school obesity prevention program for African-American girls: The Minnesota GEMS pilot study. Ethnicity \& Disease, 13(1), 54-64.

Sue, D., \& Sue, D. (2003). Counseling the culturally diverse: Theory and practice (4th ed.). Hoboken, NJ: Wiley.

Sussner, K. (2007). The influence of maternal acculturation on early childhood feeding, nutritional status and the development of obesity: A study of Latino immigrant mothers and children. (Doctoral dissertation, Harvard University, 2007). Dissertation Abstracts International, 68, Retrieved from PsycINFO database.

Talleyrand, R. M. (1998, July). The psychological impact of acculturation on people of Haitian descent: What does it mean to be Haitian-American? Paper presented at the 25th Annual Conference for the Association of Haitian Physicians Abroad, Chicago, IL.

Talleyrand, R. M. (1999, August). African American women's acculturation, racial identity, and eating disorder symptoms. Paper presented at the Annual Convention of the American Psychological Association, Boston, MA.

Ten Eyck, T. (2008). Public opinion and obesity: A different look at a modern-day epidemic. Obesity in childhood and adolescence, Vol 1: Medical, biological, and social issues (pp. 183-197). Westport, CT: Praeger/Greenwood.

Thompson, J. K. \& Smolak, L. (Eds.). (2001). Body image, eating disorders, and obesity in youth: Assessment, prevention, and treatment. Washington, DC: American Psychological Association.
Triandis, H.C. (1995). Individualism \& collectivism. Boulder, CO: Westview Press.

Villarosa, L. (1994). Body and Soul: The African American women's guide to physical health and emotional well-being. New York: Harper Perennial.

Wing, R. R., Tate, D. F., Gorin, A. A., Raynor, H. A., \& Fava, J. L. (2006). A self-regulation program for maintenance of weight loss. New England Journal of Medicine, 355(15), 1563-1571.

Yeh, C., Carter, R., \& Pieterse, A. (2004). Cultural values and racial identity attitudes among Asian American students: An exploratory investigation. Counseling and Values, 48(2), 82-95.

Zametkin, A. J., Zoon, C. K., Klien, H. W., \& Munson, S. M. (2004). Psychiatric aspects of child and adolescent obesity: A review of the past 10 years. Journal of the American Academy of Child and Adolescent Psychiatry, 43, 134-150.

Zimmerman, B. J. (1995). Self-efficacy and educational development. In A. Bandura (Ed.), Self-efficacy in changing societies (pp. 202-231). New York: Cambridge University Press.

Zimmerman, B. J. (1999). Attaining self-regulation: A social-cognitive perspective. In M. Boekaerts, P. Pintrich, \& M. Seidner (Eds.), Self-regulation: Theory, Research, and Applications. Orlando, FL: Academic Press.

Zimmerman, B. J. (2008). Investigating self-regulation and motivation: Historical background, methodological developments, and future prospects. American Educational Research Journal, 45, $166-183$. 


\section{Spirituality in Counseling and Psychotherapy The FACE-SPIRIT Model}

\section{Radha Horton-Parker and R. Charles Fawcett}

This book contains a variety of concrete integration approaches for counselors, therapists, helping professionals, instructors, and students that are both practical and flexible. The applications are categorized as either implicit (done within one's self without the client's awareness), or explicit (performed outwardly with the client). The four implicit strategies are grouped under the acronym FACE and the six explicit strategies using the acronym SPIRIT.

This textbook is designed to be complete and thorough enough to be used as a stand-alone guide for a class. However, it is short, direct, and easy enough to read to be used as a supplemental textbook addressing spirituality in counseling practice. The book covers the historical relationship between spirituality and counseling, theories of faith development and spirituality, methods for working with clients' diverse beliefs, spiritual assessment techniques, ethical issues, research findings, references, and of course the specific strategies of the FACE-SPIRT model.

\section{SPECIAL FEATURES}

- a near exhaustive review of the literature on the topic of counseling and spirituality

- vignettes and examples of counselor-client dialogue illustrating each technique

- Discussion questions at the end of each chapter to facilitate classroom dialogue and individual synthetic learning

- a companion DVD that shows the authors using these techniques with the case studies, available for sale through the American Counseling Association (www.counseling.org)

Coutents

Part One: The Why, When, and How of Integrating Spirituality Into Counseling and Psychotherapy

1 The Why, When, and How of Integrating Spirituality Into Counseling and Psychotherapy

2 When to Integrate Spirituality Into Counseling and Psychotherapy

3 An Overview of Spiritual Competencies and Ethical Practice

4 How to Integrate Spirituality Into Counseling and Therapy: The FACE-SPIRIT Model

Part Two: FACE - Implicit Strategies for Integrating Spirituality Into Counseling and Psychotherapy

$5 \quad$ Focusing on the Present

$6 \quad$ Asking for Guidance

7 Compassion Cultivation

$8 \quad$ Existential Empathy

Part Three: SPIRIT - Explicit Strategies for Integrating Spirituality Into Counseling and Psychotherapy

$9 \quad$ Simile and Metaphor

10 Prayer

11 Interpretation of Sacred Texts

12 Ritual Therapy

13 Imagery

14 Transgression Relief

15 Closing Thoughts \& Vignettes 


\section{Professional update}

American School Counselor Association

July 3-6, 2010

Boston, MA

Contact: $800-306-4722$

Website: http://www.schoolcounselor.org

\section{American Psychological Association}

August 12-15, 2010

San Diego, CA

Contact: $800-374-2721$

email: convention@apa.org

Website: http:// apa.org

\section{PERMISSIONS AND COPYRIGHT}

All rights are reserved. No part of this publication may be reproduced, photocopied, faxed, stored in a retrieval system, or transmitted in any form or by any means, electronic, mechanical, recording or otherwise, without the prior written permission of the publisher.
Back issues are available for sale. Reproduction requires permission and payment of fees. It is illegal and a violation of federal copyright law to reproduce this publication without permission. Direct all inquiries to the permissions editor. 